\title{
HOLLOW CATHODE ELECTRON GUN FOR THE EXCITATION OF CW LASERS
}

\author{
J.J. ROCCA, J. MEYER and G.J. COLLINS ${ }^{1}$ \\ Department of Electrical Engineering, Colorado State University, Fort Collins, CO 80523, USA
}

Received 20 October 1981

\begin{abstract}
Well collimated electron beam discharges with an energy of $1 \mathrm{~kW}$, at currents up to $0.1 \mathrm{~A}$, have been obtained at ambient pressures up to 0.4 Torr in Ar and 1.4 Torr in He using hollow cathode electron guns. The guns operate on a continuous or dc basis. These guns have the unique feature of providing a clear optical path through the axis. We suggest the use of such an electron beam discharge for excitation of $\mathrm{cw}$ ion lasers.
\end{abstract}

We report the generation of electron beams, with discharge powers up to $1 \mathrm{~kW}$, using hollow cathode plasma guns. In contrast with thermoionic electron guns, that operate at pressures below $10^{-4}$ Torr, and plasma guns that operate in argon below $20 \mu$ Torr [1], our guns generate an electron beam operating at ambient pressures up to 0.4 Torr in argon and up to 1.4

* Work supported by the National Science Foundation. The early portion of these studies were supported by AFOSR.

1 Alfred Sloan Foundation Fellow, 1979-81.
Torr in helium. Moreover the structure of the electron guns described below is such that they provide a clear optical path through the gun, as shown in fig. 1. This permits one to easily match the electron beam created plasma volume with the corresponding volume of an optical resonator. The combination of operating pressure in the Torr region without the need of complex differential pumping, and the ease of matching the beam plasma and optical cavity volume makes the electron gun of fig. 1 useful for attempting electron beam. excitation of $\mathrm{cw}$ lasers. It is important to note that

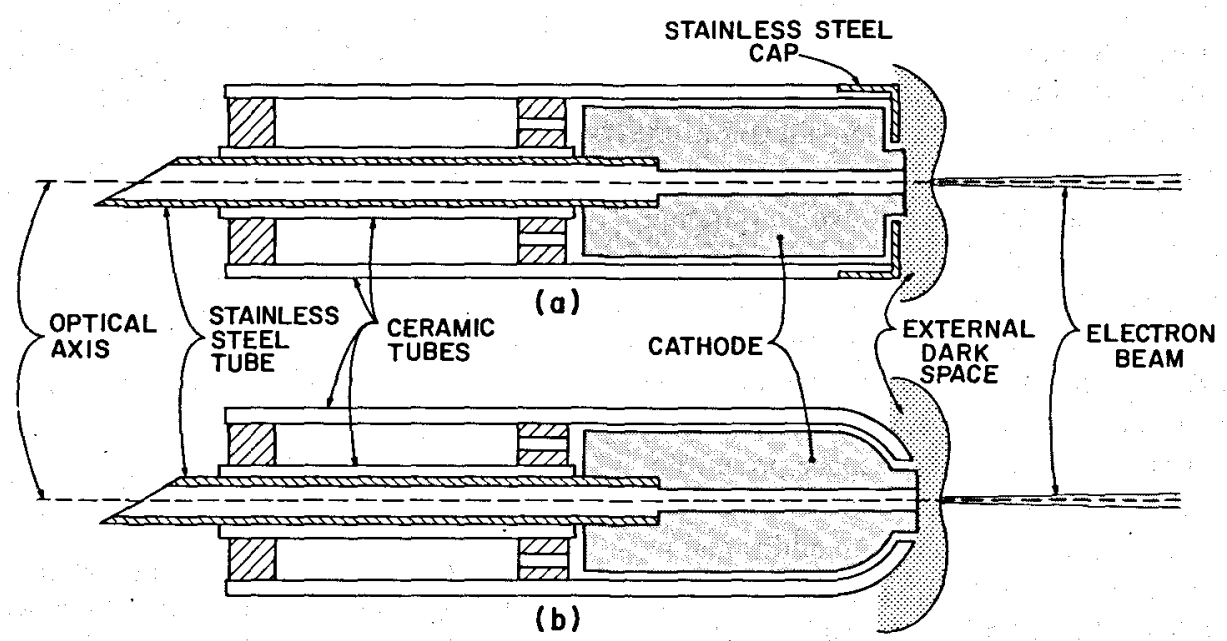

Fig. 1. (a) Hollow cathode electron gun with front surface shielded by a stainless steel cap. Electron gun inside diameter 4.7 mm, outside diameter $31 \mathrm{~mm}$. (b) Same electron gun, with front surface shielded by a ceramic tube. 
electron beam generation efficiencies of 60 to $70 \%$ have been measured in the past for hollow cathode electron guns [1].

The design of two electron guns is shown in fig. 1 . High purity graphite, that has a low sputtering yield, was selected as cathode material. To avoid emission from the cathode external walls the graphite is shielded with a ceramic tube (alumina 99.8). The distance between the cathode and the shield is approximately $1 \mathrm{~mm}$. The front wall of the gun is also partially shielded by a stainless steel piece, or by the ceramic tube, as shown in fig. $1 \mathrm{a}$ and $1 \mathrm{~b}$ respectively. This obstructed glow mode of operation is required if a well colimated electron beam is desired. The hollow cathode external diameter and the thermal characteristics of the shield determine the working cathode temperature, since the cathode is radiation cooled. The cathode was operated at negative high voltage, and the anode grounded. The position of the anode is unimportant, and does not have any influence on the electron beam characteristics as long as the distance between electrodes is kept larger than the cathode dark space.

The hollow cathode electron guns have two modes of operation. One mode is a high impedance one, in which an electron beam is produced, and the other is a low impendance mode where no electron beam is produced. In the low impedance mode the cathode operates as a regular hollow cathode [2], the voltage drop is a few hundred $V$ and a positive column occupies most of the distance between the electrodes. In both modes of operation an internal plasma created within the cathode hole, shown in fig. 1 , works as a virtual cathode for the discharge in the following manner. Electrons emitted by the internal walls of the cathode, as a result of ion bombardment and photoelectric emission, are accelerated through an internal voltage drop of a few hundred $\mathrm{eV}$. These electrons are electrostatically trapped inside the hole and lose most of their energy in ionizing and exciting collisions that sustain the internal plasma. In the high impedance mode of operation an external dark space, shown in fig. 1, also exists. This external dark space presents a voltage drop of several $\mathrm{kV}$ to the electrons which emerge from the hollow cathode plasma. An additional portion of the electron beam emission is produced by ions that bombard the cathode front wall and create secondary electrons that are subsequently accelerated through the same external dark space. Operation in the beam mode occurs over a limited range of current and pressure. When these parameters are not properly chosen the external dark space vanishes, production of the electron beam ceases and the discharge then operates in the low impedance mode. This transition is also evident in the $I-V$ characteristic of fig. 2a.

To efficiently pump gas lasers with longitudinal electron beam excitation a long active medium is required, so that the entire electron beam energy is deposited. We surround the electron beam drift tube with a solenoid capable of creating an axial magnetic field of strength up to $3.5 \mathrm{kG}$. The magnetic field helps to keep the electron beam collimated and thereby diminishes the energy lost to the walls of the plasma tube. The electron gun was placed $5 \mathrm{~cm}$ from the end of the solenoid, where the magnetic field strength is 250 G. The magnetic field is observed to increase the plasma density and as a consequence the ion flux to the cathode. This results in enhanced secondary emission of electrons from the cathode and a lower discharge impedance (see the dashed curves in fig. 2).
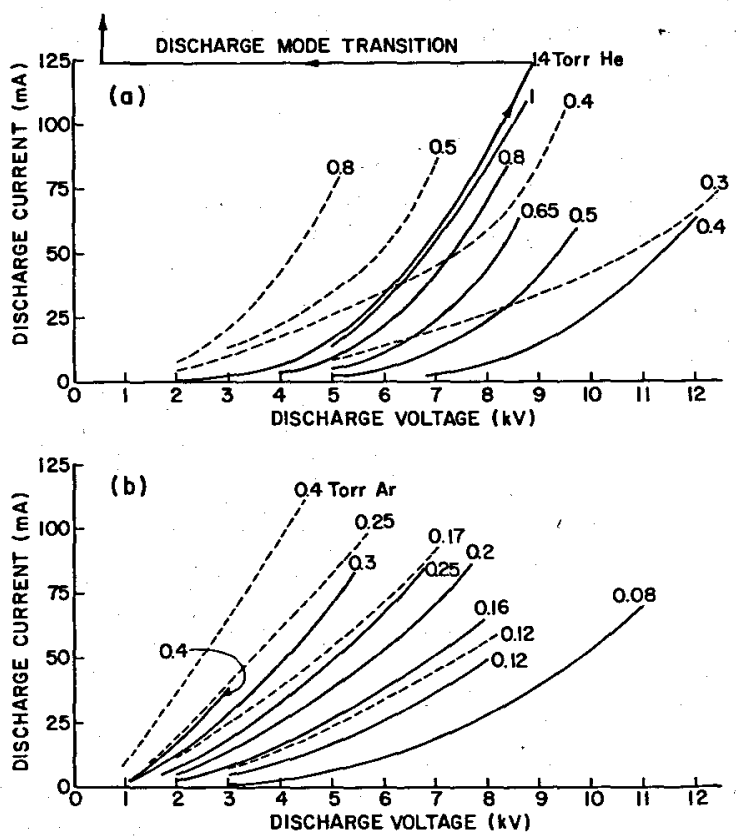

Fig. 2. (a) $I-V$ characteristics of the electron beam discharge in helium. (b) $I-V$ characteristics of the electron beam discharge in argon. - without magnetic field, - - - operating the gun in the fringing field of a $3.5 \mathrm{kG}$ solenoid. Field strength at the cathode front face; $250 \mathrm{G}$. 


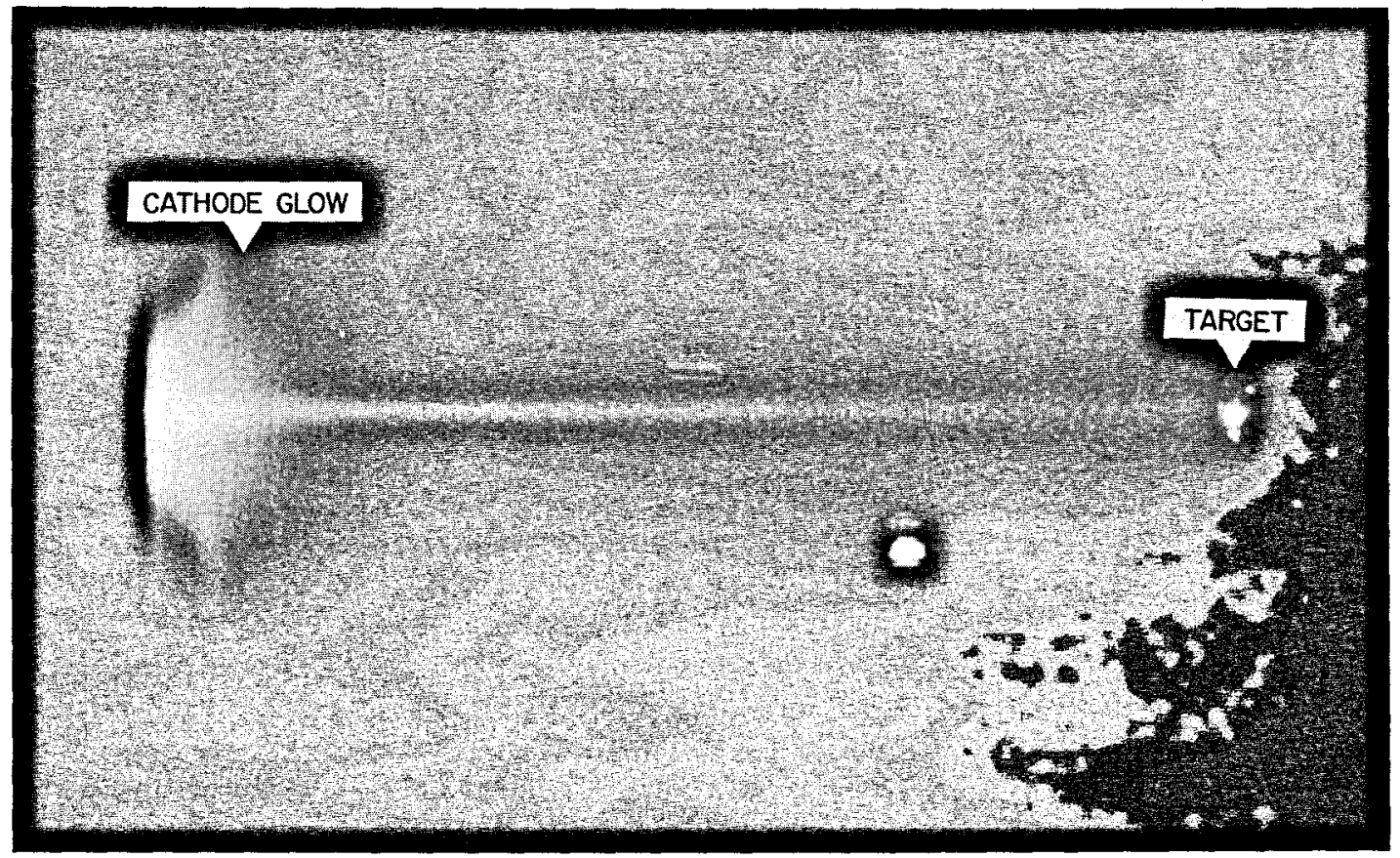

Fig. 3. Electron beam created in helium at 0.4 Torr without applied magnetic field.

The $I-V$ characteristics of the hollow cathode electron guns with and without applied magnetic field are shown in fig. 2 . The transition from the beam mode to the low impedance mode is only shown in the plot corresponding to a 1.4 Torr helium discharge in this figure but occurs for all curves at the maximum discharge power shown. Fig. 3 shows an electron beam produced in helium at a pressure of 0.4 Torr.

In summary, well collimated electron beams with energies of several hundred $W$ have been generated with hollow cathode electron guns at pressures that are typical for the operation of $\mathrm{cw}$ gas lasers. The electron guns are sturdy, of simple construction and provide an optical path through the center axis. One suggested use of this electron gun is excitation of $\mathrm{cw}$ ion lasers with the electron beam created plasmas as an active medium.

\section{References}

[1] K.L. Boring and L.A. Stauffer, A new nonthermoionic electron gun, Proc. Nat. Electron. Conf. 19 (1963) 535.

[2] C.W. Willet, Introduction to gas lasers, population inversion mechanisms (Pergamon Press, 1974). 